\title{
Pyruvate Dehydrogenase Complex Deficiency Due to PDHA1 Mutation-A Rare Treatable Cause for Episodic Ataxia in Children
}

\author{
Harini Pavuluri ${ }^{1} \cdot$ Alfiya $^{2} \cdot$ Ramshekhar N. Menon $^{2} \cdot$ Sruthi S. Nair $^{1} \cdot$ Soumya Sundaram ${ }^{3}(0)$
}

Received: 3 November 2021 / Accepted: 10 December 2021 / Published online: 8 February 2022

(c) Dr. K C Chaudhuri Foundation 2022

To the Editor: Episodic or recurrent cerebellar ataxia in children is rare, and neurometabolic conditions are an important cause [1]. Here, we present a young boy with episodic ataxia due to pyruvate dehydrogenase complex (PDC) deficiency with good response to thiamine supplementation.

A 5-y-old-boy, born of nonconsanguineous parentage, presented to us with three episodes of fever-triggered ataxia since $3 \mathrm{y}$ of age. Each episode lasted for 2-7 d. On examination during the third event, he had impaired tandem gait, left upper limb incoordination and bilateral optic disc pallor. The child was completely normal interictally. He had unremarkable birth and developmental histories and family history was noncontributory.

His serum and cerebrospinal fluid lactate were elevated, while other metabolic parameters were normal. MRI brain revealed symmetric T2-weighted and fluid-attenuated inversion recovery hyperintensity in the bilateral dentate nuclei without any white matter or corpus callosum involvement. Clinical exome sequencing revealed a pathogenic hemizygous 4-base-pair duplication in exon 12 (c.1273_1276dup) of the PDHAl gene (frameshift mutation). He was initiated on high-dose thiamine, levocarnitine, and alpha lipoic acid following which he had only one minor episode in the next $3 \mathrm{y}$.

Soumya Sundaram

ssdr.soumya@gmail.com

1 Department of Neurology, Sree Chitra Tirunal Institute for Medical Sciences and Technology, Thiruvananthapuram, Kerala, India

2 R Madhavan Nayar Center for Comprehensive Epilepsy Care, Department of Neurology, Sree Chitra Tirunal Institute for Medical Sciences and Technology, Thiruvananthapuram, Kerala, India

3 Pediatric Neurology and Neurodevelopmental Disorders, Department of Neurology, Sree Chitra Tirunal Institute for Medical Sciences and Technology, Thiruvananthapuram, Kerala 695011, India
Involvement of corpus callosum, grey matter nuclei in brainstem, basal ganglia, thalamus, dentate nucleus, and ventriculomegaly are the neuroimaging findings reported in PDC deficiency [2, 3]. The diagnostic clues in this patient were elevated lactate and dentate nucleus involvement [2, 4]. However, isolated dentate nucleus involvement has not been reported yet. Usually, frameshift mutations are reported to cause more severe and fatal manifestations of PDC deficiency as compared to missense mutations, but our patient had a mild phenotype. Treatment options include high-dose thiamine, alpha lipoic acid, ketogenic diet, and dichloroacetate [2, 3]. PDC deficiency merits consideration in a child with episodic ataxia, since it is eminently treatable.

\section{Declarations}

Conflict of Interest None.

\section{References}

1. Pavone $\mathrm{P}$, Praticò AD, Pavone V, et al. Ataxia in children: early recognition and clinical evaluation. Ital J Pediatr. 2017;43:6.

2. Pavlu-Pereira H, Silva MJ, Florindo C, et al. Pyruvate dehydrogenase complex deficiency: updating the clinical, metabolic and mutational landscapes in a cohort of Portuguese patients. Orphanet J Rare Dis. 2020;15:298.

3. Patel KP, O’Brien TW, Subramony SH, Shuster J, Stacpoole PW. The spectrum of pyruvate dehydrogenase complex deficiency: clinical, biochemical and genetic features in 371 patients. Mol Genet Metab. 2012;105:34-43.

4. Imbard A, Boutron A, Vequaud C, et al. Molecular characterization of 82 patients with pyruvate dehydrogenase complex deficiency. Structural implications of novel amino acid substitutions in E1 protein. Mol Genet Metab. 2011;104:507-16.

Publisher's Note Springer Nature remains neutral with regard to jurisdictional claims in published maps and institutional affiliations. 